\title{
Avaliação do desempenho de sistema de irrigação por gotejamento em cultivo de maracujá
}

\author{
Performance evaluation of drip irrigation system in passion fruit cultivation
}

Evaluación del rendimiento del sistema de riego por goteo en el cultivo de fruta de la pasión

Antônio Ricardo Santos de Andrade

ORCID: https://orcid.org/0000-0003-1384-0888

Universidade Federal do Agreste de Pernambuco, Brasil

E-mail: ricoarsa@gmail.com

Edijailson Gonçalves da Silva

ORCID: https://orcid.org/0000-0002-1436-1718

Universidade Federal do Agreste de Pernambuco, Brasil

E-mail: edijailsongoncalves@gmail.com

Edes Torres da Silva

ORCID: https://orcid.org/0000-0002-1686-663X Universidade Federal do Agreste de Pernambuco, Brasil

E-mail: edestorres@gmail.com

Rodrigo Gomes Pereira

ORCID: https://orcid.org/0000-0001-9635-4126 Universidade Federal do Agreste de Pernambuco, Brasil

E-mail: rgpereira2005@hotmail.com

Jennifer Ferreira da Silva

ORCID: https://orcid.org/0000-0001-9350-9268

Universidade Federal Rural de Pernambuco, Brasil

E-mail: jennycat14y@gmail.com

Claudia Machado Costa

ORCID: https://orcid.org/0000-0001-7761-7963

Universidade Federal do Agreste de Pernambuco, Brasil

E-mail: claudiamacosta3@gmail.com

Valentin Rubén Orcón Zamora

ORCID: https://orcid.org/0000-0001-6010-5806

Universidade Federal Rural de Pernambuco, Brasil E-mail: vorcon2871@gmail.com

Adiel Felipe da Silva Cruzb

ORCID: https://orcid.org/0000-0002-8571-6125

Universidade Federal Rural de Pernambuco, Brasil E-mail: felipe.adiel@gmail.com

Maria Emanuely da Silva Ferreira

ORCID: https://orcid.org/0000-0003-4221-8318

Universidade Federal do Agreste de Pernambuco, Brasil

E-mail: emanuely.darck@gmail.com

Maria Gorete dos Santos Silva

ORCID: https://orcid.org/0000-0002-5569-3540

Universidade Federal do Agreste de Pernambuco, Brasil

E-mail: goretesantos_sj@outlook.com

Danilo Araújo de Noronha

ORCID: https://orcid.org/0000-0002-5668-2982

Universidade Federal do Agreste de Pernambuco, Brasil

E-mail: danilo_2018@gmail.com

\section{Resumo}

A constante avaliação do desempenho dos sistemas de irrigação torna-se necessária para verificar se as condições previstas no projeto condizem com o observado em campo. Desta forma, objetivou-se avaliar o desempenho de sistemas de irrigação localizada visando a indicar as medidas de uniformidade. O trabalho foi conduzido na Fazenda Areião, área experimental da ESALQ/USP, Piracicaba/SP, cultivada com maracujá. A avaliação baseou-se nos seguintes coeficientes de desempenho: coeficiente de uniformidade de Christiansen (CUC), coeficiente de uniformidade de distribuição (CUD), coeficiente de uniformidade estatístico (CUE) e eficiência de aplicação (Ea) dos dados de distribuição de volume de água obtidos de teste realizado em campo. No presente trabalho os resultados mostraram que CUC (89,86\%), CUD (90,37\%) e CUE (99,88\%) estão dentro dos padrões de classificação de 
desempenho dos emissores e eficiência média de aplicação foi de 83,03\%, indicando que a avaliação de desempenho do sistema de irrigação por gotejamento, encontra-se em perfeitas condições de operação.

Palavras-chave: Localizada; Avaliação; Irrigação.

\begin{abstract}
The constant evaluation of the performance of the irrigation systems is necessary to verify if the conditions foreseen in the project are consistent with the observed in the field. Thus, the objective was to evaluate the performance of localized irrigation systems in order to indicate uniformity measures. The work was carried out at Fazenda Areião, an experimental area of ESALQ/USP, Piracicaba/SP, cultivated with passion fruit. The evaluation was based on the following performance coefficients: Christiansen's uniformity coefficient (CUC), distribution uniformity coefficient (CUD), statistical uniformity coefficient (CUE) and application efficiency (Ea) of the volume distribution data of water obtained from field tests. In the present work, the results showed that CUC (89.86\%), CUD (90.37\%) and CUE $(99.88 \%)$ are within the standards of performance classification of the emitters and the average application efficiency was $83.03 \%$, indicating that the performance evaluation of the drip irrigation system is in perfect operating condition.

Keywords: Localized; Evaluation; Irrigation.
\end{abstract}

\title{
Resumen
}

La constante evaluación del desempeño de los sistemas de riego se hace necesaria para verificar si las condiciones previstas en el proyecto coinciden con el observado en campo. De esta forma, se objetivó evaluar el desempeño de sistemas de riego localizado para indicar las medidas de uniformidad. El trabajo fue realizado en la Hacienda Areião, área experimental de la ESALQ/USP, Piracicaba/SP, cultivada con maracuyá. La evaluación se basó en los siguientes coeficientes de rendimiento: coeficiente de uniformidad de Christiansen (CUC), coeficiente de uniformidad de distribución (CUD), coeficiente de uniformidad estadística (CUE) y eficiencia de aplicación (Ea) los datos de distribución del volumen de agua obtenidos de las pruebas realizadas sobre el terreno. En el presente trabajo los resultados mostraron que CUC (89,86\%), CUD (90,37\%) y CUE (99,88\%) están dentro de los estándares de clasificación de desempeño de los emisores y eficiencia media de aplicación fue de 83,03\%, indicando que la evaluación de desempeño del sistema de riego por goteo, se encuentra en perfectas condiciones de operación.

Palabras clave: Localizada; Evaluación; Irrigación.

\section{Introdução}

Os sistemas de irrigação localizados por gotejamento são sistemas de altamente eficientes, capazes de reduzir as perdas de água. E na escolha de um método de irrigação, além de todos os fatores de seleção do próprio sistema e método, deve-se ter como base os parâmetros de uniformidade que está relacionada à engenharia hidráulica do sistema e, principalmente, os parâmetros da eficiência do sistema na qual envolve aspectos agronômicos da cultura. Sendo assim, é importante a avaliação dos parâmetros que afetam a qualidade da irrigação, especialmente aqueles relacionados à uniformidade de distribuição de água, sendo essencial no manejo de irrigação visando manter a eficiência de aplicação de água dos sistemas, em razão da possibilidade de entupimento de emissores causados por partículas minerais e orgânicas presentes no solo (Silva \& Silva, 2005; Levien \& Figueirêdo, 2013; Alves et al., 2020).

No sistema de irrigação localizada por gotejamento, o emissor responsável pela aplicação de água é o gotejador, que, de acordo com Salomão (2012), aplica água ao solo em gotas, em um ponto e diretamente sobre a zona radicular da planta, com pequenas vazões. Os gotejadores segundo Bernardo et al. (2006) são as peças principais do sistema de irrigação por gotejamento, sua função é dissipar a pressão disponível na linha lateral e aplicar vazões pequenas e constantes.

No entanto, para que a irrigação seja eficiente, é imperativo que os sistemas exibam alta uniformidade de aplicação da água. Uma vez instalado um projeto de irrigação, é necessário verificar se as condições inicialmente previstas se confirmam no campo. Para isso, devem ser avaliadas as condições de pressão, vazão e lâminas de água aplicadas. Melhorar a uniformidade de um sistema de irrigação é uma das decisões mais importantes para o manejo adequado da água aplicada, pois o excesso de água, além da perda de água, pode transportar nutrientes para profundidades do solo não exploráveis pelas raízes (Bernardo et al., 2006; Júnior et al., 2016). Em caso de aplicação deficitária, podem ocorrer prejuízos na produção, principalmente nos períodos críticos (Júnior et al., 2016). 
Dentre os sistemas de irrigação utilizados nas atividades agrícolas, a irrigação por gotejamento tem-se destacado principalmente pela alta uniformidade de aplicação de água, alcançando assim alta eficiência de aplicação. Essas características de desempenho propiciam outra vantagem do uso da irrigação por gotejamento, ou seja, a aplicação de produtos químicos através da água de irrigação (Souza et al., 2006). Para se avaliar as condições em que o sistema de irrigação está operando, parâmetros de desempenho devem ser definidos com base em determinações de campo, como vazão, uniformidade de distribuição de aplicação de água, eficiência de aplicação de água, percentagem da área adequadamente irrigada e do tempo de irrigação.

Um dos principais parâmetros utilizados na avaliação de um sistema de irrigação é a uniformidade de aplicação de água sobre a área irrigada, que está associado à eficiência de irrigação. A irrigação desempenha um papel importante no aumento da produtividade das culturas e a uniformidade de distribuição de aplicação de água pelos emissores tem sido um fator limitante para se obter um manejo eficiente do uso da água aplicada. Uma vez que, a uniformidade de distribuição de aplicação indica como a água está sendo distribuída na área e se as plantas estão recebendo as mesmas quantidades equivalentes de água. Assim, estudos visando aumentar a eficiência do uso da água nesta atividade agrícola tornam-se fundamentais (Frizzone et al., 2012; Oliveira, 2014). Em projetos de irrigação, quando o sistema está devidamente dimensionado, uma boa uniformidade de distribuição é essencial, pois é a garantia de que todas as plantas receberão aproximadamente a mesma quantidade da solução aplicada.

A eficiência de irrigação é um conceito amplamente utilizado, tanto em projetos quanto no manejo de sistemas de irrigação. De acordo com Keller e Bliesner (1990) citado por Souza et al. (2006), o conceito de eficiência engloba dois aspectos básicos: a uniformidade de aplicação e as perdas, que podem ocorrer durante a operação do sistema. Para Frizzone e Dourado Neto (2003), as medidas de eficiência quantificam fisicamente a qualidade da irrigação, por incorporarem algumas consequências da uniformidade; Se por um lado, as medidas de uniformidade dependem apenas do grau de dispersão com que a água é aplicada, por outro lado, as medidas de eficiência dependem tanto da uniformidade como da forma como sistema da irrigação é operado. A baixa uniformidade de distribuição de água reduz a eficiência de aplicação de água e a produtividade (Júnior et al., 2016).

Para que a eficiência de aplicação de água pelo sistema de irrigação atingir valores elevados, é necessário que as perdas durante a operação sejam os menores possíveis e maiores a uniformidade de distribuição e aplicação de água pelos emissores seja maior. De acordo com Silva et al. (2004) o desempenho da irrigação pode ser determinado por um índice de uniformidade ou coeficientes que expresse a variabilidade espacial da água aplicada, sendo o Coeficiente de Uniformidade de Christiansen (CUC) o mais utilizado. O Coeficiente de Uniformidade de Distribuição (CUD) ou simplesmente uniformidade de distribuição (UE) e o Coeficiente de Uniformidade Estatístico (CUE) também são usados em uma escala menor. Quando esses coeficientes são maiores ou iguais um determinado valor arbitrário, os coeficientes de uniformidade de distribuição da água aplicada pelos emissores é considerada aceitável (Silva \& Silva, 2005). Keller e Karmeli (1974) citados por Zocoler (2021) classificam os valores de CUC como excelentes quando maiores que $90 \%$, bons na faixa de $85 \%$ a $90 \%$, regulares entre $70 \%$ e $80 \%$ e ruins quando menores que $70 \%$. Enquanto o CUD é considerado excelente quando superior a $84 \%$, bom entre 68 e $84 \%$, regular entre 52 a $68 \%$ e ruim inferior a 32\%. Para CUE, é considerado excelente quando ultrapassa $90 \%$, bom entre 80 e $90 \%$, regular entre $70-80 \%$ e ruim menos que $60 \%$.

A eficiência de aplicação $(\mathrm{Ea})$ refere-se à relação entre o volume de água disponível para a cultura e o volume aplicado pelo emissor. De acordo com Frizzone e Dourado Neto (2003) o parâmetro de eficiência expressa a qualidade da irrigação e, ao mesmo tempo, incorporam algumas consequências da uniformidade, que expressa a variabilidade do volume de aplicação de água dos emissores na superfície do solo, onde a eficiência depende tanto da uniformidade quanto dos fatores puramente agronômicos, como quantidade e frequência de irrigação, características físicas do solo, profundidade da raiz, 
superfície molhada, permeabilidade do solo.

A análise da distribuição espacial da água aplicada em áreas irrigadas é essencial na implementação de estratégias adequadas de manejo da irrigação. Para isso, é fundamental determinar os coeficientes de uniformidade de distribuição de água na avaliação do desempenho de sistemas de irrigação (Silva et al., 2006). Diante do exposto, após a instalação do sistema de irrigação na área cultivada com maracujá, foi necessário verificar se as condições previstas no projeto condizem com a realidade do campo. Assim, o presente trabalho tem como objetivo estudar, por meio de ensaio em campo, avaliar os parâmetros de uniformidade de distribuição de aplicação de água de um sistema de irrigação localizada, do tipo gotejamento, em cultivo de maracujá.

\section{Metodologia}

\section{Caracterização da área experimental}

A avaliação do sistema de irrigação por gotejamento foi conduzida na Fazenda Areião, área experimental da ESALQ/USP, Piracicaba, SP. A fazenda está localizada no município de Piracicaba, São Paulo, cujas coordenadas geográficas

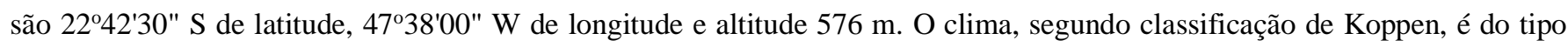
Cwa, ou seja, clima subtropical úmido com estiagem no inverno, com precipitação pluviométrica média de $1247 \mathrm{~mm}$, temperatura de $21,1^{\circ} \mathrm{C}$ e umidade relativa média de $74 \%$, velocidade do vento de $2,2 \mathrm{~m} \mathrm{~s}^{-1}$.

O solo da área onde se encontra o sistema de irrigação localizada é classificado como Terra Roxa Estruturada (Alfisol), com uma declividade média de 2,3. A área total irrigada é de $3.024 \mathrm{~m}^{2}$, sendo que a unidade operacional de $1.512 \mathrm{~m}^{2}$ é constituída por 12 linhas laterais, com espaçamento de 3,5 m e 108 plantas distribuídas nessas 12 linhas (9 plantas por linha). Ao longo da linha lateral havia nove pontos de emissão, cada um com dois emissores separados $0,5 \mathrm{~m}$. A cultura instalada no campo experimental foi o maracujá, com dois emissores por planta (ponto de emissão) cujas plantas apresentam o espaçamento de 3,5 x 4 m entre fileiras e entre plantas na mesma fileira, respectivamente.

\section{Determinação da uniformidade do sistema de irrigação}

Para avaliação da uniformidade de distribuição de água aplicada do sistema de irrigação por gotejamento, foi utilizado seguindo a metodologia de Keller e Karmeli (1975) que recomendam que a coleta das vazões em quatro emissores ao longo de quatro linha lateral. Desta forma, o conjunto de dados utilizado na avaliação de desempenho do sistema de irrigação foi constituído por16 valores de vazão por emissores, que consiste da coleta de dados da seguinte forma: a determinação da vazão de 32 emissores (16 x 2 emissores por plantas), distribuídos em quatro tubulações laterais. Para isso, pode-se seguir o seguinte critério consistem em selecionar 4 linhas laterais ao longo da linha de derivação: a primeira linha lateral, a segunda situada a $1 / 3$ do comprimento da linha de derivação, a terceira a 2/3 da linha de derivação e a última linha lateral 3/3, seguindo o mesmo critério, selecionam-se 16 emissores na unidade operacional, sendo quatro em cada lateral, ou seja, a primeira será a mais próxima do ponto de alimentação da lateral, a segunda a $1 / 3$ do comprimento da linha lateral, a terceira a 2/3 do comprimento da linha lateral e a última da linha lateral a 3/3. Em cada ponto de emissão foram determinadas as vazões de água dos emissores cujo tempo estabelecido para coleta foi de 5 min determinado com auxílio de um cronômetro de precisão e de uma proveta graduada em $\mathrm{mL}$ e a vazão de cada emissor foi de $40 \mathrm{~cm}^{3} \mathrm{~min}^{-1}\left(2,40 \mathrm{Lh}^{-1}\right)$ sob uma pressão de serviço de $10 \mathrm{mca}$. e o coeficiente de variação de fabricação (CVF) tipo auto-compensante e marca carborundum foi de $5 \%$.

A vazão ou volume de aplicação de água pelos emissores (Va) foi determinada pelo método volumétrico, que segundo Salomão (2008), para coleta de volume pequeno, pode ser utilizada de acordo com a Equação 1: 


$$
\mathrm{Q}=\mathrm{V}_{\mathrm{a}}=\frac{\mathrm{V}_{\mathrm{c}} \div 1000}{\mathrm{~T} \div 60}
$$

Em que: $\mathrm{Q}=\mathrm{V}_{\mathrm{a}}=$ vazão ou volume de aplicação de água pelos emissores $\left(\mathrm{L} \mathrm{h}^{-1}\right) ; \mathrm{V}_{\mathrm{c}}=$ volume de água coletado pelos emissores (m L) e $\mathrm{t}=$ tempo de coleta em (min).

A partir das vazões de água coletadas em campo de dois emissores por planta distribuídos em quatro tubulações laterais (ponto de emissão) na unidade operacional em estudo, sendo que os valores totais da vazão de água por ponto de emissão foram obtidos a partir da soma dos valores de vazão de água dos dois emissores, do qual foram se torna a base para a determinação os parâmetros de uniformidade de irrigação das distribuições superficiais da água aplicada: coeficiente de uniformidade de Christiansen (CUC), coeficiente de uniformidade de distribuição (CUD) e coeficiente de uniformidade estatístico (CUE) segundo as metodologias:

\section{a) Metodologia de Christiansen (1942)}

Bralts et al. (1988) citado por Mantovani et al. (2009), mencionam que muitos métodos têm sido propostos para se estimar a uniformidade de distribuição da água pelos emissores a campo, dentre os quais o mais utilizado o Coeficiente de Uniformidade de Christiansen do sistema (CUC) utilizou-se a seguinte equação:

$$
\text { CUC }=100\left(1-\frac{\sum_{\mathrm{i}=1}^{\mathrm{n}}\left|\mathrm{Q}_{\mathrm{i}}-\mathrm{Q}_{\text {med }}\right|}{\mathrm{n} \mathrm{Q}_{\text {med }}}\right)
$$

Em que: $\mathrm{CUC}=$ Coeficiente de uniformidade de distribuição de água pelos emissores de Christiansen (\%); $\mathrm{Q}_{\mathrm{i}}=\mathrm{V}_{\mathrm{ai}}=$ vazão ou volume de aplicação de água pelos emissores por cada gotejador $\left(\mathrm{L} \mathrm{h}^{-1}\right)$; $\mathrm{Q}_{\text {med }}=$ média das vazões coletadas de todos os gotejadores $\left(\mathrm{L} \mathrm{h}^{-1}\right) ; \mathrm{n}=$ número de gotejadores analisados.

b) Metodologia de Merrian e Keller (1978)

Para a determinação do Coeficiente de Uniformidade de Distribuição de água pelos emissores (CUD) proposta por Merrian e Keller (1978), que é baseada na razão entre as vazões mínimas e médias dos emissores, pela seguinte equação:

$$
\mathrm{CUD}=\frac{\mathrm{Q}_{25 \%}}{\mathrm{Q}_{\text {med }}} 100
$$

Em que: CUD = Coeficiente de uniformidade de distribuição de água pelos emissores, (\%); Q $25 \%=$ média de 25\% do total de gotejadores com as menores vazões $\left(\mathrm{L} \mathrm{h}^{-1}\right)$; $\mathrm{Q}_{\text {med }}=$ média das vazões coletadas nos gotejadores na subárea, $\left(\mathrm{Lh}^{-1}\right)$. Nos sistemas de irrigação por gotejamento a Eq. (03) é conhecida com a uniformidade de distribuição (CUD), a qual tem sido denominada uniformidade de emissão (UE). 
b) Metodologia da ASAE (1996)

O Coeficiente de Uniformidade Estatística (CUE) tem sido outro termo utilizado para a avaliação dos sistemas de irrigação localizada. Segundo Bralts et al. (1987) citado por Mantovani et al. (2009), o coeficiente de uniformidade estatística foi apresentado primeiramente por Wilcox e Swailes (1947) para a avaliação de sistemas de irrigação. Tal como estabelecido na época, o coeficiente de uniformidade estatística baseou-se no coeficiente de variação, para o qual se aceitam valores acima de $75 \%$, conforme a seguinte expressão:

$$
\mathrm{CUE}=(1-\mathrm{CVq})=\left(1-\frac{\mathrm{S}}{\mathrm{Q}_{\text {med }}}\right) 100
$$

Em que: CUE = coeficiente de uniformidade estatística (\%); CVq - coeficiente estatística de variação da vazão dos emissores (adimensional); $\mathrm{S}=$ desvio padrão da vazão dos emissores $\left(\mathrm{Lh}^{-1}\right) ; \mathrm{Q}_{\mathrm{med}}=$ média das vazões coletadas nos gotejadores na subárea, $\left(\mathrm{Lh}^{-1}\right)$.

O coeficiente estatístico de variação de vazão dos emissores (CVq) foi determinado pelo desvio-padrão dos valores de vazão coletados em relação à vazão média e representa uma medida de dispersão relativa dos dados e, quando aplicado em uma amostra de emissores novos, é definido como o coeficiente de variação de fabricação, o qual representa pequenas diferenças construtivas de cada emissor (Solomon, 1979). Neste trabalho, todos os emissores avaliados apresentaram coeficiente de variação inicial de vazão igual 5\%. Para verificação do grau de aceitabilidade dos parâmetros dos Coeficientes de uniformidades irrigação CUD e CUE como para o CVq, usou neste trabalho o critério segundo de Merrian e Keller (1978) e da ASAE (1996).

\section{Eficiência de aplicação de água pelo sistema de irrigação}

A partir dos resultados valores das uniformidades encontradas para unidade operacional em estudo, determinou-se os parâmetros de eficiência de aplicação de água (Ea) de irrigação por gotejamento por meio da metodologia baseia-se na expressão matemática padrão da ABNT (1985) e da ASAE (1996). Segundo (Bernardo et al., 2006; Mantovani et al., 2009) os métodos tradicionais de avaliação da eficiência de um sistema de irrigação localizada que refletem a realidade de campo, são determinados pelos seguintes parâmetros, expressos da forma abaixo:

- Eficiência de aplicação (Ea)

$$
\mathrm{E}_{\mathrm{a}}=\frac{\mathrm{V}_{\mathrm{s}}}{\mathrm{V}_{\mathrm{t}}}
$$

Onde: $\mathrm{E}_{\mathrm{a}}=$ eficiência de aplicação, em \%

A eficiência do sistema de irrigação também é obtida conforme a equação descrita por San Juan (1993):

$$
\mathrm{E}_{\mathrm{a}}=\text { Cra } \mathrm{x} \text { CUD }
$$

Onde: CUD = coeficiente de uniformidade de distribuição, em \%; Cra = relação entre água transpirada e água aplicada a planta (decimal). 
Para a interpretação dos valores obtidos pela CUD utilizou-se a metodologia de Merrian e Keller (1978), onde o CUD maior que $90 \%$ classifica-se como excelente; entre $80 \%$ e $90 \%$, bom; $70 \%$ e $80 \%$, regular; e menor que $70 \%$ é ruim (Silva et al., 2017). Segundo San Juan (1993) os valores de "Cra" estão compreendidos entre 0,8 e 0,9, e em condições especiais podem ser atingidos os valores de 0,95 e em teoria pode-se alcançar valor igual a 1,0, mas este último é possível apenas em regiões úmidas em que a irrigação por gotejamento é utilizada como apoio, e não em regiões bastante áridas que se empregam frequentemente o gotejamento. Em razão disto adotou-se o valor de "Cra" igual a 0,9 (Silva et al., 2017).

- Eficiência de armazenamento (Es)

$$
\mathrm{E}_{\mathrm{S}}=\frac{\mathrm{V}_{\mathrm{S}}}{\mathrm{V}_{\mathrm{r}}}
$$

- Eficiência de distribuição (Ed)

$$
E_{d}=\frac{V_{s}}{V_{s}+V_{p}}
$$

- Eficiência em potencial de aplicação (Epa)

$$
E_{p a}=\frac{V_{s}+V_{p}}{V_{e v}+V_{v}+V_{p}+V_{s}}
$$

- Percolação profunda (Pp)

$$
\mathrm{P}_{\mathrm{p}}=\frac{\mathrm{V}_{\mathrm{p}}}{\mathrm{V}_{\mathrm{t}}}
$$

- Percentagem de déficit $(\mathrm{Gd})$

$\mathrm{Gd}=100-\mathrm{E}_{\mathrm{s}}$

Em que: $\mathrm{V}_{\mathrm{t}}=$ Volume total de água aplicada à parcela; $\mathrm{V}_{\mathrm{s}}=$ Volume de água armazenada na profundidade do sistema radicular; $\mathrm{V}_{\mathrm{r}}=$ Volume de água necessário para suprir o déficit hídrico; $\mathrm{V}_{\mathrm{ev}}=$ Volume total de água evaporada; $\mathrm{V}_{\mathrm{v}}=$ Volume de água derivado pelo vento; $\mathrm{V}_{\mathrm{d}}=$ Volume de déficit, sendo igual a $\left(\mathrm{V}_{\mathrm{r}}-\mathrm{V}_{\mathrm{s}}\right) ; \mathrm{V}_{\mathrm{p}}=$ Volume total de água percolado abaixo das raízes; $\mathrm{V}_{\mathrm{e}}=$ Volume total de água escoado para fora da parcela irrigada, em $\mathrm{mL}$.

Para o sistema de irrigação por gotejamento podem ser desprezadas as variáveis $\mathrm{V}_{\mathrm{ev}}, \mathrm{V}_{\mathrm{v}}$ e $\mathrm{V}_{\mathrm{e}}$; obtém-se que $\mathrm{E}_{\mathrm{pa}}=$ $100 \%$ e $E_{d}=E_{a}$. $O$ volume total de água por ponto de emissão $\left(V_{\text {total }}\right)$ pode ser estimado pela expressão:

$$
\mathrm{V}_{\text {total }}=\mathrm{q} \mathrm{tn}
$$

Em que: $\mathrm{q}$ = vazão do gotejador, $\mathrm{em} \mathrm{cm}^{3} \mathrm{~min}^{-1} ; \mathrm{t}=$ tempo aplicação de água, em min; $\mathrm{n}=$ número de gotejadores por ponto de emissão.

\section{Ensaios de campo na obtenção do volume de água coletado pelos emissores}

No estudo os volume de água aplicados pelo emissores $\left(\mathrm{V}_{\mathrm{a}}\right)$ e do volume de água coletado pelos emissores $\left(\mathrm{V}_{\mathrm{c}}\right)$ para a avaliação da irrigação por gotejamento em campo, onde cada coletor representa uma área equivalente a 3,5 x 4 m e área total 
de 36 x 42 m. Nesta situação, a fração de área correspondente a cada coletor é igual a 0,00925 (0,925\% da área molhada), ou seja, representa a relação entre a área de uma malha de $14 \mathrm{~m}^{2}$ e a área da unidade operacional avaliada de $1.512 \mathrm{~m}^{2}$.

$\mathrm{Na}$ área experimental do estudo com objetivo de avaliar do sistema de irrigação por gotejamento, foram usados dois emissor por planta, ao todo foram 36 coletores, conforme metodologia de Keller \& Karmeli (1975) para a avaliação da uniformidade de distribuição e da eficiência de água aplicada pelo sistema de irrigação, de forma que cada coletor representa uma fração de área correspondente 1/36 avos da área avaliada, ou seja, 0,027 (0,27 \% da área molhada). A água dos emissores foi coletada com auxílio de 36 recipientes coletores posicionados abaixo dos gotejadores, apoiados sobre um suporte de madeira, o qual teve como objetivo permitir o manuseio de todos os copos de uma única vez, fazendo com que todos os emissores das linhas avaliadas fossem coletados juntamente, a fim de evitar possíveis erros na estabilização e aferição da pressão desejada. Os volumes de água coletadas foram mensurados com o auxílio de um cronômetro e de uma proveta de plástico de volume de $100 \mathrm{~mL}$ graduada com precisão de $2 \mathrm{~mL}$.

\section{Resultados e Discussão}

\section{Determinação da uniformidade de emissão do sistema de irrigação por gotejamento}

A partir das vazões de água coletadas pelo emissores obtidos dos testes realizados em campo durante 5 minutos, com dois emissores por planta, em que, a vazão de cada emissor (q) é igual $40 \mathrm{~cm}^{3} \mathrm{~min}^{-1}$ (2,40 $\left.\mathrm{Lh}^{-1}\right)$, os valores totais da vazão por ponto de emissão foram obtidos a partir da soma dos valores de vazões de dois emissores, cujos resultados se encontram na Tabela 1. Saber que a variação da vazão nos emissores ao longo das linhas laterais, faz-se necessário transformar volume de água coletados pelos emissores $\left(\mathrm{V}_{\mathrm{c}}\right)$ obtidos de teses realizados em campo, em unidades de vazão (mL), para valores de volume de aplicação de água pelos emissores $\left(\mathrm{V}_{\mathrm{a}}\right)$, em unidade de $\mathrm{cm}^{3} \mathrm{~min}^{-1} \mathrm{ou} \mathrm{Lh}^{-1}$ que normalmente é usada para irrigação localizada.

Tabela 1. Valores dos volumes de água coletados pelos emissores $\left(\mathrm{V}_{\mathrm{c}}\right)$ no tempo de 5 minutos, e dos volumes de aplicação de água pelos emissores $\left(\mathrm{V}_{\mathrm{a}}\right)$ obtidos dos testes de realizados no campo para avaliação do sistema de irrigação por gotejamento em cultivo de maracujá.

\begin{tabular}{|c|c|c|c|c|c|}
\hline \multirow[t]{2}{*}{ Lateral } & \multirow[t]{2}{*}{ Pontos de emissão } & \multicolumn{2}{|c|}{$\begin{array}{c}\text { Volume de água coletado em cada } \\
\text { emissor (mL) }\end{array}$} & \multicolumn{2}{|c|}{$\begin{array}{c}\text { Volume de aplicação de água pelos } \\
\text { emissores (Va) }\end{array}$} \\
\hline & & Emissor 1 & Emissor 2 & $\left(\mathrm{~cm}^{3} \min ^{-1}\right)$ & $\left(\mathrm{Lh}^{-1}\right)$ \\
\hline \multirow{4}{*}{ Início 1/1 } & Primeiro $1 / 1$ & 176 & 171 & 69.40 & 4,16 \\
\hline & Segundo $1 / 3$ & 209 & 152 & 72.20 & 4,33 \\
\hline & Terceiro $2 / 3$ & 198 & 211 & 81.80 & 4,90 \\
\hline & Último $3 / 3$ & 166 & 155 & 64.20 & 3,85 \\
\hline \multirow{4}{*}{ Segunda 1/3 } & Primeiro 1/1 & 210 & 242 & 90.40 & 5,42 \\
\hline & Segundo $1 / 3$ & 223 & 226 & 89.80 & 5,38 \\
\hline & Terceiro $2 / 3$ & 221 & 209 & 86.00 & 5,16 \\
\hline & Último $3 / 3$ & 204 & 190 & 76.80 & 4,72 \\
\hline \multirow{4}{*}{ Terceira $2 / 3$} & Primeiro $1 / 1$ & 209 & 219 & 85.80 & 5,13 \\
\hline & Segundo $1 / 3$ & 199 & 244 & 88.60 & 5,31 \\
\hline & Terceiro $2 / 3$ & 150 & 217 & 73.40 & 4,40 \\
\hline & Último $3 / 3$ & 221 & 216 & 87.40 & 5,24 \\
\hline \multirow{4}{*}{ Final 3/3 } & Primeiro $1 / 1$ & 241 & 244 & 97.00 & 5,82 \\
\hline & Segundo $1 / 3$ & 248 & 238 & 97.20 & 5,83 \\
\hline & Terceiro $2 / 3$ & 247 & 255 & 100.40 & 6,02 \\
\hline & Último $3 / 3$ & 205 & 190 & 79.00 & 4.74 \\
\hline
\end{tabular}

Fonte: Andrade et al. (2021). 
As vazões máxima (Qmáx), mínima (Qmín) e média (Qméd) observadas para o sistema foram respectivamente 100,40; 64,20 e 83,83 $\mathrm{cm}^{-3}$ min (Tabela 2), estando a Qméd observada abaixo do valor mencionado pelo fabricante de 80 $\mathrm{cm}^{3} \mathrm{~min}^{-1}$ (40 $\mathrm{cm}^{-3} \min \times 2$ emissores por planta). Esses resultados podem ser justificado em razão da baixa pressão de serviço utilizada no momento do teste, entupimento de emissores, pela topografia do terreno e pelas características hidráulicas do sistema. Segundo Scaloppi e Dias (1996) avaliar a variabilidade da lâmina de água aplicada no solo tem por objetivo reduzir os custos de energia, água e perda de nutrientes, além de proporcionar uma melhor condição de suprimento hídrico às culturas irrigadas. Segundo Boman (1989) citado por Sousa et al. (2011) o estudo da variabilidade funciona como um indicador da desigualdade (ou igualdade) da distribuição de água no campo.

Com base nos valores da Tabela 1, calculou-se os parâmetros de uniformidade Coeficiente de uniformidade de distribuição de água pelos emissores de Christiansen (CUC), uniformidade de distribuição de água (CUD), coeficiente estatístico de variação de vazão dos emissores $(\mathrm{CVq})$ e o coeficiente de uniformidade estatística (CUE) com a finalidade de avaliar a performance do sistema de irrigação por gotejamento, cujos valores foram 89,86\%, 90,37 \%, 12,47\% e 99,88 \%, respectivamente (Tabela 2). Segundo os critérios de classificação de desempenho hidráulico dos emissores, os valores de CVq e CUC, CUD e CUE indicam que o sistema é adequado, que se situa na categoria aceitável para sistemas de irrigação localizada, e indicam que o sistema está funcionando de forma adequada e Segundo Reis et al., (2005) esses resultados traduzem o bom estado de conservação dos gotejadores e uma ótima condição de pressão e vazão.

Tabela 2. Valores das vazões máxima (Qmáx), mínima (Qmín) e média (Qméd), Coeficiente de uniformidade de distribuição de Christiansen (CUC), coeficiente de uniformidade de distribuição de água (CUD), coeficiente de variação de variação de vazão dos emissores (CVq) e o coeficiente de uniformidade estatística (CUE) do sistema de irrigação por gotejamento em cultivo de maracujá.

\begin{tabular}{ccccccc}
\hline Qmáx & Qmín & Qméd & CUC & CUD & CVq & CUE \\
\hline$-{ }_{-100,40}$ & 64,20 & 83,83 & 89,86 & 90,37 & 12,47 & 99,88 \\
\hline
\end{tabular}

Fonte: Andrade et al. (2021).

O CVq obtido de 0,1247 (12,47\%) é classificado como médio segundo Salomon (1979). O autor cita que valores < $3 \%$ é considerado excelente; $3-7 \%$ médio; $10-14 \%$ pobre e > 14\% inaceitável. O CUE encontrado no presente estudo de 98,88\% está dentro do recomendado conforme a norma da ASAE (1996) de 85\% a 90\%, em cultivos anuais em zonas áridas e topografia uniforme (Silva et al., 2017). Segundo Pizarro (1996) a CUD é um indicador dos problemas distribuição da irrigação. Uniformidade alta é importante para irrigação em solos arenosos, onde a redistribuição lateral da água é limitada. Excessos como os valores obtidos neste trabalho, resultam frequentemente em lâmina percolada e lixiviação de nutrientes para fora da zona radicular.

Segundo Martín-Benito (1993) a uniformidade de distribuição de água de instalações novas situa-se próximo de 90\%, podendo diminuir consideravelmente com o uso continuado. Por isso, um valor de CUD de $80 \%$ é mais realista, sendo que os valores de eficiência podem ser menores devido a problemas de manejo. Para o autor, no momento da realização de um projeto de irrigação localizada, os fatores econômicos contribuirão para determinar a seleção da CUD, devendo-se comparar o custo (e possibilidade) do maior consumo de água com a maior inversão inicial da instalação. Entretanto segundo Neto et al., (2013), valores de coeficiente de uniformidade e de distribuição inferiores a 90\% em sistemas localizados, só podem ser admitidos se a precipitação pluvial tem um valor significativo durante o cultivo, ou se a diminuição dos custos do sistema com a redução da uniformidade compensar a diminuição da receita devido à redução na produção da cultura. 
O coeficiente estatístico de variação de vazão dos emissores (CVq) que indica a variação devido a causas diferentes da hidráulica, decorrente da variação de fabricação, obstrução do emissor, tempos do uso do sistema e variação da vazão dos emissores, em função dos fatores construtivos e temporais. O valor encontrado para o CVq (12,47\%), foi considerado médio, caracterizando bom coeficiente de variação das vazões dos emissores instalados no campo, porém se observou entupimento de alguns emissores com pouco uso do sistema de irrigação. Segundo normas da ASAE (1996) quando ocorre obstrução dos emissores, o CVq apresenta valores acima de 0,20. Como o resultado apresentou valor superior, infere-se, que a causa da obstrução, ocorrida pela presença de material sólido (mineral ou orgânico) na água de irrigação passou pelos filtros. Também, dada a pequena magnitude de fatores causadores de desuniformidade (perdas de carga, declividade e diferencial de temperatura da água) e uma vez determinado o valor do coeficiente de variação de fabricação (CVf) no início do experimento (5\%) e CVq no decorrer do experimento $(12,47 \%)$ as diferenças encontradas foram relacionadas ao processo de entupimento de alguns dos emissores. De acordo com Wu (1995) para valores de CUE maiores que $70 \%$ e CVq próximo de 0,3, a hipótese de normalidade é aceita, ou seja, é possível ajustar-se uma distribuição normal aos dados de Volume de aplicação de água dos emissores e, com isto, confirma-se que aplicação do método estatístico na avaliação das eficiências é válida.

\section{Avaliação da eficiência do sistema de irrigação por gotejamento}

Utilizando os dados da Tabela 1 e com base no valor do volume de água requerida pela cultura ou volume de irrigação real necessário para a cultura de $175 \mathrm{~cm}^{3} \cdot$ planta $^{-1} \cdot \mathrm{min}^{-1}$, calculou-se o volume de aplicação $\left(\mathrm{V}_{\mathrm{a}}\right)$, o volume de armazenamento $\left(V_{\mathrm{s}}\right)$, o volume percolado $\left(\mathrm{V}_{\mathrm{p}}\right)$ e o volume de déficit $\left(\mathrm{V}_{\mathrm{d}}\right)$ em litros por planta dia, onde os valores encontrados estão ilustrados na Tabela 3 .

Tabela 3. Volumes de água apliacada $\left(V_{a}\right)$, armazenad $\left(V_{s}\right)$, percolada $\left(V_{p}\right)$ e de déficit $\left(V_{d}\right)\left(\mathrm{cm}^{3} \cdot \operatorname{planta}^{-1} \min ^{-1}\right)$ para o tempo de coleta de 5 minutos no sistema de irrigação por gotejamento em cultivo de maracujá.

\begin{tabular}{|c|c|c|c|c|c|}
\hline \multirow[b]{2}{*}{ Lateral } & \multirow[b]{2}{*}{ Ponto de emissão } & \multicolumn{4}{|c|}{ Volume de água } \\
\hline & & $\begin{array}{c}\text { Aplicado } V_{\mathrm{a}} \\
\left(\mathrm{cm}^{3} \cdot \text { planta }^{-1} \mathrm{~min}^{-1}\right)\end{array}$ & $\begin{array}{c}\text { Armazenado } V_{s} \\
\left(\mathrm{~cm}^{3} \cdot \text { min }^{-1}\right)\end{array}$ & $\begin{array}{c}\text { Percolado } V_{p} \\
\left(\mathrm{~cm}^{3} \cdot \mathrm{min}^{-1}\right)\end{array}$ & $\begin{array}{l}\text { Déficit } V_{d} \\
\left(\mathrm{~cm}^{3} \cdot \mathrm{min}^{-1}\right)\end{array}$ \\
\hline \multirow{4}{*}{ Início 1/1 } & Primeiro $1 / 1$ & 347.00 & 347.00 & 0.00 & 3.00 \\
\hline & Segundo $1 / 3$ & 452.00 & 350.00 & 102.00 & 0.00 \\
\hline & Terceiro $2 / 3$ & 428.00 & 350.00 & 78.00 & 0.00 \\
\hline & Último $3 / 3$ & 485.00 & 350.00 & 135.00 & 0.00 \\
\hline \multirow{4}{*}{ Segunda 1/3 } & Primeiro $1 / 1$ & 361.00 & 350.00 & 11.00 & 0.00 \\
\hline & Segundo $1 / 3$ & 449.00 & 350.00 & 99.00 & 0.00 \\
\hline & Terceiro $2 / 3$ & 443.00 & 350.00 & 93.00 & 0.00 \\
\hline & Último $3 / 3$ & 486.00 & 350.00 & 136.00 & 0.00 \\
\hline \multirow{4}{*}{ Terceira 2/3 } & Primeiro $1 / 1$ & 409.00 & 350.00 & 59.00 & 0.00 \\
\hline & Segundo $1 / 3$ & 430.00 & 350.00 & 80.00 & 0.00 \\
\hline & Terceiro $2 / 3$ & 367.00 & 350.00 & 17.00 & 0.00 \\
\hline & Último $3 / 3$ & 502.00 & 350.00 & 152.00 & 0.00 \\
\hline \multirow{4}{*}{ Final 3/3 } & Primeiro 1/1 & 321.00 & 321.00 & 0.00 & 29.00 \\
\hline & Segundo $1 / 3$ & 394.00 & 350.00 & 44.00 & 0 \\
\hline & Terceiro $2 / 3$ & 437.00 & 350.00 & 87.00 & 0 \\
\hline & Último $3 / 3$ & 395.00 & 350.00 & 45.00 & 0 \\
\hline Total & & 6706.00 & 5568.00 & 1138.00 & 32 \\
\hline
\end{tabular}

Fonte: Andrade et al. (2021).

A partir dos dados da Tabela 3, os parâmetros de eficiência referentes à avaliação do sistema de irrigação por gotejamento usando-se critérios matemático padrão ou tradicional (CMP) foram calculados, tomando por base, ainda, os resultados desses valores, que claramente delimita as áreas que definem a eficiência de aplicação de água do sistema de 
irrigação, os parâmetros de eficiência referentes à avaliação do sistema de irrigação por gotejamento de maneira analítica foram determinados, através das Equações de 5 a 1, considerando-se para o cálculo os valores do volume aplicado pelos dois emissores para o tempo de coleta de 5 minutos. Os resultados são apresentados de maneira resumida os valores de Ea, Es, Pp e Gd observadas para sistema foram respectivamente, $83,03 \%, 99,43 \%, 16,97 \%$ e $0,57 \%$, conforme descritos na Tabela 4 .

Tabela 4. Parâmetros de avaliação de eficiência do sistema irrigação por gotejamento em cultivo de maracujá, através do MMP. Fonte: Andrade et al. (2021).

\begin{tabular}{lcccc}
\hline Parâmetros de eficiência do sistema de irrigação & Ea (\%) & Es (\%) & Pp (\%) & Gd (\%) \\
\hline Método matemático tradicional (padrão) & $-16,97$ & 0,57 \\
\hline
\end{tabular}

Fonte: Autores.

Para a eficiência de aplicação ou distribuição de água do sistema do presente estudo (Ea), obteve-se valor de 83,03\%, valor este que estão dentro dos padrões de classificações de desempenho dos emissores para um sistema de irrigação por gotejamento de acordo com ASAE (1996). A eficiência de armazenamento (Es = 99,43\%), perdas por percolação $(\mathrm{Pp}=$ 16,97\%), grau de déficit $(\mathrm{Gd}=0,57 \%)$. Segundo Silva et al. (2004) a área adequadamente irrigada é uma operação na qual se busca o ponto ótimo entre o porcentual de área que deve receber aplicações de água em quantidades iguais ou superiores à lâmina requerida pela cultura para satisfazer exigências de produtividade, com o menor porcentual de excesso possível.

$\mathrm{O}$ excesso de água aplicada representa um aumento no consumo de energia, reduzindo a margem de lucro do produtor, com possíveis impactos na lixiviação de nutrientes e no meio ambiente. Por outro lado, a inadequação na distribuição incorreta da água, que pode determinar baixos valores de eficiência de irrigação, pode levar a efeitos desfavoráveis como: baixa produtividade por área; baixa produtividade por unidade de água aplicada; diminuição da área total irrigada; efeitos prejudiciais ao meio ambiente e menor lucratividade com a agricultura irrigada (Schons, 2010).

Considerando-se a eficiência de aplicação de água em uma área de irrigação, observa-se que, de toda a água aplicada de $83,03 \%$ permanecem úteis às plantas, sendo que $16,97 \%$ constituíram perdas por percolação profunda. A eficiência de armazenamento indica que o reabastecimento da zona radicular foi de 99,43\%, com um déficit de apenas 0,57\%. Também se pode notar que a eficiência de armazenamento foi elevada, indicando que ocorreu excesso de irrigação, resultando em considerável perda por percolação. Entretanto, a Es e a Pp foram elevados, indicando alto armazenamento de água na zona radicular e grande volume de água perdida por percolação profunda. Os resultados obtidos neste estudo corroboram com os obtidos por Neto et al., (2013) e Silva (2010) no sistema de irrigação por gotejamento.

De acordo com Frizzone (1992) citado por Rocha et al. (1999), o coeficiente de uniformidade de distribuição de água e a eficiência de aplicação são os principais parâmetros utilizados nesta avaliação, pois expressam a qualidade da irrigação e são decisivos no planejamento e operação desses sistemas. O coeficiente de uniformidade expressa a variabilidade da lâmina de irrigação na superfície do solo e é influenciado principalmente pelo espaçamento entre aspersores, velocidade do vento e pressão de serviço. A eficiência da aplicação de água em uma área irrigada expressa a relação percentual entre o volume de água efetivamente disponibilizado à planta, na profundidade de efetividade de seu sistema radicular, aqui denominado de volume útil, e o volume total de água aplicado pela irrigação (Silva et al., 2002).

Verifica-se que, o valor da Ea é adequado para um método de irrigação por gotejamento, cujo é superior $80 \%$ ao recomendado pela ASEA (Keller \& Bliesner, 1990), sendo que o ideal seria superior à 90\%. Esses resultados podem ser justificado em razão da baixa pressão de serviço utilizada no momento do teste, entupimento de emissores, pela topografia do terreno e pelas características hidráulicas do sistema e provavelmente pelos valores atípicos também denominados outliers, 
representa um caso que difere substancialmente da maioria dos dados, que pode ser vista em gráficos de Q-Q plot e P-P plot normal, e que causa redução nos parâmetros da eficiência do sistema de irrigação (Figuras 1 e 2). Esta eficiência se aproximou a de Silva (2010), que avaliou sistema de irrigação de gotejamento no semiárido de Pernambuco com eficiência de aplicação média de $83,73 \%$.

Figura 1. Diagrama Q-Q plot para avaliação da normalidade e outliers dos valores do volume de água aplicado pelos emissores $\left(\mathrm{V}_{\mathrm{a}}\right)$ no sistema irrigação por gotejamento em cultivo de maracujá.

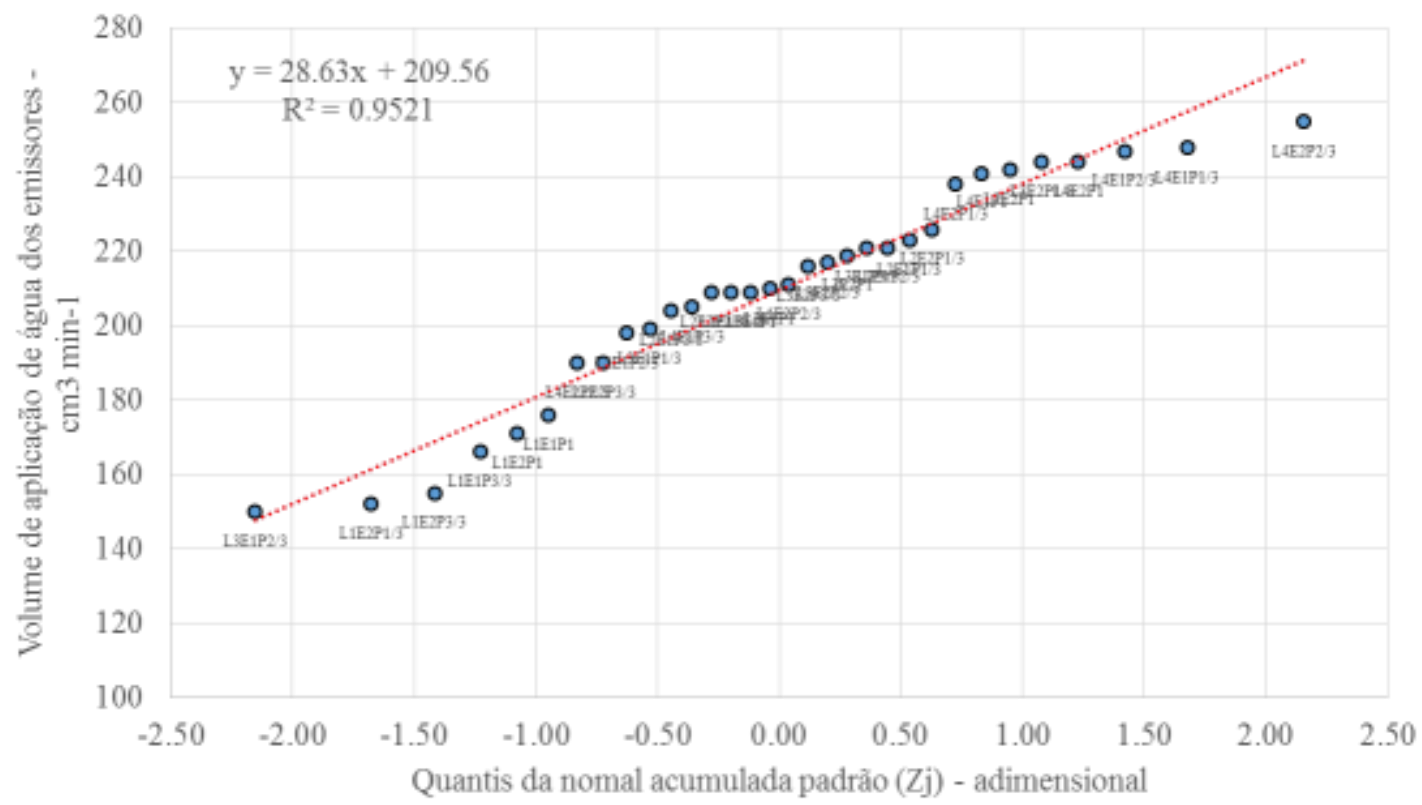

Notação: LEP - L = representa as linhas de gotejo ao logo da derivação do sistema de irrigação (4 linhas: L1, L2 e L3); E = número de emissores por planta (2 emissores: E1 e E2) e P = Posição dos emissores na linha de gotejo (4 posições: P1, P1/2 e P3/3). Fonte: Andrade et al. (2021).

Verifica-se pelo diagrama Q-Q plot existem pontos poucos dispersos e bem alinhados em torno da equação linear (os pontos seguem um padrão linear), com valor alto de coeficiente de determinação $\left(\mathrm{R}^{2}\right)$ de 0,952 ao nível de significância de 1 \%, evidenciando a normalidade dos dados observados (Figura 1). De acordo com Hartmann et al. (2011), a avaliação da normalidade da distribuição dos dados é essencial para a adequada descrição e caracterização da amostra, e na análise inferencial, além de identificar grandes assimetrias, descontinuidades dos valores de $\mathrm{V}_{\mathrm{a}}$.

Segundo Daniel (1976) citado por Nóbrega (2010) os efeitos dos pontos marcados fora da reta, por não seguirem a mesma configuração dos demais pontos, são julgados como pontos de emissão significativos em termos de diferença entre valores de Va de cada ponto de coleta, ou seja, se eles entre si diferem estatisticamente (Figura 4). Os pontos com efeitos ou diferenças significantes positivos são marcados acima da reta, ao passo que aqueles negativos são marcados abaixo desta. Como se pode ver pelo referido gráfico Q-Q plot, a existência clara de diferença significativa entre os valores de volume de água aplicado pelos emissores $\left(\mathrm{V}_{\mathrm{a}}\right)$ nos seguintes pontos de emissão (pontos de coleta): L1E2P1/3, L1E2P3/3, L4E1P1/3 e L4E2P2/3, ou seja, pontos esses que apresentação diferença nos valores de $\mathrm{V}_{\mathrm{a}}$ em comparação com os demais, o qual explica o valor encontrado para o $\mathrm{CVq}(12,47 \%)$, que para o sistema de irrigação localizado é caracterizando como valor médio, causando variação das vazões dos emissores, e consequentemente redução na uniformidade de distribuição da água aplicada as plantas.

Ainda pelo gráfico Q-Q plot (Figura 1), observa-se espaços ou lacunas abertas em torno da origem e ao final da curva 
dos valores de quantis, denominado "gaps", indicando a presença de valores discrepantes nos dados. Esses valores discrepantes influenciar na uniformidade de distribuição e na eficiência de aplicação da água reduzindo os valores dos parâmetros de eficiência do sistema de irrigação. Segundo Hund et al., (2002) a identificação de valores discrepantes no gráfico de probabilidade Q-Q plot é mais perceptível a visualizar a abertura (gaps) em torno das caudas da distribuição, na origem e no final dos dados.

A estatística do diagrama P-P plot da Figura 2, corrobora a afirmação anterior (Q-Q plot) mostrando que há normalidade dos dados observados. De acordo com Hund et al., (2002) no diagrama P-P plot, os pontos plotados devem recair em uma reta de $45^{\circ}$ de inclinação entre $(0,0)$ e $(1,1)$.

Pela Figura 2, claramente, é possível identificar visivelmente que um pequena quantidades de pontos estão acima e próximo da reta de $45^{\circ}$ de inclinação (diagonal) sem desvios fortes e pouquíssimos pontos abaixo, mostrado uma leve assimetria à direita da distribuição, e não compromete a aceitação (confirmação) da hipótese de normalidade dos dados observados.

Figura 2. Diagrama P-P plot para avaliação da normalidade e outliers dos valores do volume de água aplicado pelos emissores $\left(\mathrm{V}_{\mathrm{a}}\right)$ no sistema irrigação por gotejamento em cultivo de maracujá.

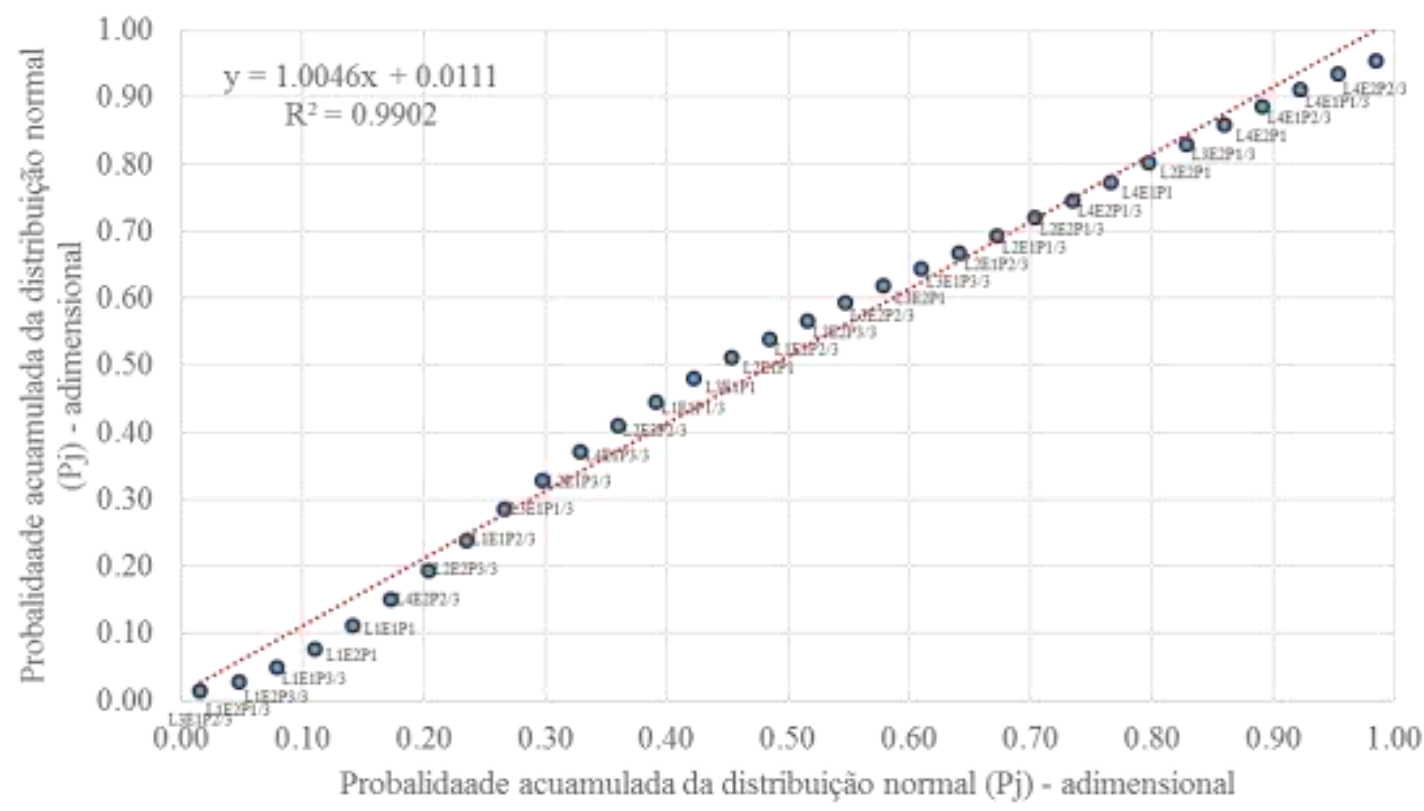

Notação: LEP - L = representa as linhas de gotejo ao logo da derivação do sistema de irrigação (4 linhas: L1, L2 e L3); E = número de emissores por planta (2 emissores: E1 e E2) e P = Posição dos emissores na linha de gotejo (4 posições: P1, P1/2 e P3/3). Fonte: Andrade et al. (2021).

No gráfico de probabilidade P-P plot mostrados na Figura 2, parece não haver indicação de que haja algum diferença significativo nos dados. Como se pode ver pelo gráfico, que não há indicação da presença de valores discrepantes. Os gaps observados nos gráficos da Figura 2, embora consideravelmente pequenos, indicam esta mesma conclusão observada no gráfico Q-Q plot, há presença de valores discrepantes nos dados.

\section{Conclusão}

Com base nas discussões referentes aos resultados apresentados neste trabalho, conclui-se que: (i) Os coeficientes de uniformidade de Christiansen (CUC), de uniformidade de distribuição (CUD) de uniformidade estatístico (CUE) e coeficiente 
estatístico de variação de vazão dos emissores $(\mathrm{CVq})$ foram classificados como satisfatório na área irrigada pelo sistema de irrigação localizada com a cultura de maracujá; (ii) Os resultados encontrados dos parâmetros de eficiência de aplicação de água estão dentro dos padrões de classificação de desempenho dos emissores para o sistema de irrigação localizada por gotejamento; (iii) A eficiência de armazenamento foram relativamente altos, indicando, respectivamente, excesso de irrigação, resultando em considerável perda por percolação e redução das eficiências de aplicação.

A produtividade agrícola em áreas irrigadas depende de uma série de fatores, incluindo técnicas de dimensionamento e manejo para melhorar a aplicação de água via sistemas de irrigação. Com o aumento da demanda por sistemas de irrigação por parte de maior área agricultável na região Nordeste do Brasil, destaca-se a necessidade de novas pesquisas na área, a fim de determinar o desempenho das áreas irrigadas e o nível de manejo adequado da irrigação, como seguro contra secas ou veranicos. Além disso, é fundamental avaliar os sistemas periodicamente para minimizar as perdas de água e fertilizantes. Isso se deve ao fato de que a prática da irrigação por diferentes produtores é realizada com a ausência de critérios técnicos, ocasionando perdas ou excessos na aplicação da água, sendo necessária a utilização de técnicas de manejo que visem melhorar a uniformidade da aplicação na área irrigada, capaz de reduzir as perdas de água e aumentar a produtividade.

\section{Referências}

ABNT - Associação Brasileira de Normas Técnicas (1985). Sistema de irrigação localizada: avaliação do desempenho, 85 p.

Alves, D. K. M., Teixeira, M. B., Cunha, F. N., Filho, F. R. C., Soares, J. A. B. \& Gomes, L. F. (2020). Grau de entupimento em um sistema de irrigação localizada submetido a diferentes tempos de funcionamento. Research, Society and Development, 9(8), 1-25.

Anyoji, H. \& Wu, I. P. (1994). Normal Distribution Water Application for Drip Irrigation Schedules. Transactions of ASAE, 37(1), 159-164.

ASAE - American Society of Agricultural Engineers (1996). Standard engineering practicesdata: EP458. Field evaluation of microirrigation systems. St. Joseph: ASAE, 972-797.

Bernardo, S., Soares, A. A. \& Mantovani, E. C. (2006). Manual de irrigação. (8a ed.), UFV.

Christiansen, J. E. (1942) Irrigation by sprinkling. Berkely: Universit of California.

Frizzone, J. A. \& Dourado Neto, D. D. (2003). Avaliação de sistema de irrigação. In: Miranda, J. H., Pires, R. C. de M. (ed.). Irrigação. $573-651$.

Frizzone, J. A., Freitas, P. S., Rezende, R. \& Faria, Manoel A. (2012). Microirrigação: gotejamento e microaspersão. Eduem, 356p.

Hartmann, M., Moala, F. A. \& Mendonça, M. A. (2011). Estudo das Precipitações Anuais Máximas em Presidente Prudente. Revista Brasileira de Meteorologia, 24, 561-568.

Hund, E., Massart, D. L. \& Smeyers-Verbeke, J. (2002). Robust regression and outlier detection in the evaluation of robustness tests with different experimental designs. Analytica Chimica Acta, 463, 53-73.

Júnior, M. V., Rocha, J. P. A., Demontiêzo, F. L. L. Lima, L. S. S., Carvalho, C. M. C. \& Carvalho, L. L. S. (2016). Análise comparativa de metodologia de coleta de dados para avaliação de sistemas de irrigação localizada. Revista Brasileira de Agricultura Irrigada, 10(5), $965-975$.

Karmeli, D. (1978). Estimating sprinkler distribution patterns using linear regression. Transactions of the American Society of Agricultural Engineers, 21(4), 682-686.

Keller, J. \& Bliesner, D. R. (1990). Sprinkle and trickle irrigation. von Nostrand Reinhold.

Keller, J. \& Karmeli, D. (1975). Trickle irrigation design. S.1: Rain Bird Sprinkler Manufacturing Corporation.

Levien, S. L. A. \& Figueirêdo, V. B. (2013). Metodologia simplificada para a estimativa em campo da uniformidade de sistemas de irrigação por gotejamento superficial. Revista Brasileira de Agricultura Irrigada, 7 (5), 90-299.

Mantovani, E. C., Bernardo, S. \& Palaretti, L. F. (2009). Irrigação: princípios e métodos. (2a ed.), UFV.

Martín-Benito, J. M. T. (1993). La aplicación del agua el riego y su evaluación. In: Olalla Mañas, F. M. S. Agronomia del riego. Ediciones Mundi-Prensa, $615-694$.

Merrian, J. L. \& Keller, J. (1978). Farm irrigation system evaluation: A guide for management. Logan: Agricultural and Irrigation Engineering Department, Utah State University.

Neto, J. D., Azevedo, C. A. V., Silva, L. F. D., Silva, P. F. \& Santos, C. S. (2013). Desempenho de sistema de irrigação por gotejamento em áreas de pequenos produtores do semiárido paraibano. Enciclopédia Biosfera, Centro Científico Conhecer, Goiânia, 9(16), 2013. 
Nóbrega, M. P. (2010). Estudo comparativo de gráficos de probabilidade normal para análise de experimentos fatoriais não replicados. Dissertação de Mestrado em Matemática Aplicada e Estatística, Universidade Federal do Rio Grande do Norte, Natal, RN, 196p.

Oliveira, F. C. (2014). Produtividade da água no cultivo de brócolis de cabeça sob diferentes sistemas de irrigação. Dissertação de Mestrado em Engenharia Agrícola, Universidade Federal da Grande Dourado - UFGD, 59p.

Pizarro, F. (1996). Riego localizados de alta frecuencia. (2a ed.), Ediciones Mundi Prensa.

Reis, E. F., Barros, F. M., Campanharo, M. \& Pezzopane, J. E. M. (2005). Avaliação do desempenho de sistemas de irrigação por gotejamento. Engenharia na Agricultura. 2(2), 74-81.

Rocha, E. M. M., Costa, R. N. T., Mapurunga, S. M. S. \& Castro, P. T. (1999). Uniformidade de distribuição de água por aspersão convencional na superfície e no perfil do solo. Revista Brasileira de Engenharia Agrícola e Ambiental, 3(2), 154-160.

Rocha, S. S. (2016). Estudo das Variáveis na Extração de Cobalto em um Sistema Níquel-Cobalto Utilizando Análise de Variância. Monografia (Curso de Especialização em Estatística com ênfase em Indústria e Mercado), Universidade Federal de Minas Gerais, Belo Horizonte - BH, 40p.

Salomão, L. C. (2008). Uniformidade do sistema de irrigação. In: Souza. T. R., Villas Boas, R. L., \& Saad. J. C. C. Aspectos práticos da fertirrigação. Botucatu: FEPAF, cap. 3, 13-17.

Salomão, L. C. (2012). Calibração de tanques evaporímetros de baixo custo sob diferentes diâmetros em ambiente protegido. Tese doutorado, Universidade Estadual Paulista "Júlio De Mesquita Filho, UNESP, 74p.

Salomon, K. (1979). Manufacturing variation of trickle emitters. Transactions of the ASAE, St. Joseph, 22(5), 1034-1038.

San Juan, J. A. M. (1993). Riego por goteo: teoria y práctica. $3^{\mathrm{a}}$ ed, revisada y ampliada. Ediciones Mundi-Prensa, 86-87.

Scaloppi, J. E. \& Dias, K. F. S. (1996). Relação entre a pressão de operação e a uniformidade de distribuição de água de aspersores rotativos por impacto. In: Congresso Brasileiro De Engenharia Agrícola, 26, 1996, Bauru. Resumos... Bauru: Sociedade Brasileira de Engenharia Agrícola, CD Rom.

Schons, R. L. (2010). Critérios para o aumento do índice de eficiência nos sistemas de irrigação tipo pivô central. Tese de Doutorado, Universidade Federal de Santa Maria, UFSM, RS, 139p.

Silva, C. A. \& Silva, C. J. (2005). Avaliação de uniformidade em sistemas de irrigação localizada. Revista Científica Eletrônica de Agronomia, 4(.8), 33-48.

Silva, E. M., Azevedo, J. A. \& Lima, J. E. F. W. (2002). Análise de desempenho da irrigação. Embrapa Cerrados, 84 p. - (Documentos / Embrapa Cerrados),

Silva, E. M., Lima, J. E. F. W., Azevedo, J. A. \& Rodrigues, L. N. (2004). Proposição de um modelo matemático para a avaliação do desempenho de sistemas de irrigação. Pesquisa Agropecuária Brasileira, 39, 741-748.

Silva, E. M., Lima, J. E. F. W., Rodrigues, L. N. \& Azevedo, J. A. (2006). Comparação de modelos matemáticos não-lineares empregados na análise de desempenho de sistemas de irrigação. Pesq. agropec. bras., Brasília, 41(6), 1049-1052.

Silva, E. M., Pereira, L. S., Ferreira, J. O. P., Lima, C. J. G. S. \& Silva, J. B. L. (2017). Avaliação de um sistema de irrigação por gotejamento em cultivo de quiabeiro. In: XLVI Congresso Brasileiro de Engenharia Agrícola - CONBEA 2017, Maceió, AL, Brasil, 1-4.

Silva, J. J. N. (2010). Avaliação de sistema de irrigação localizada por gotejamento no agreste de Pernambuco. In: X Jornada de ensino, pesquisa e extensão -JEPEX 2010.

Solomon, K. (1979). Manufacturing variation of trickle emitters. Transactions of the ASAE. St Joseph, 22, $1034-1038$.

Sousa, A. C. M., Sousa, F. F. P., Santos, L. N. S., Elaiuy, M. L. C., Ferrarezi, R. S., \& Matsura, E. E. (2011). Avaliação do desempenho de sistema de irrigação por gotejamento superficial em cultivo de laranja. In: XX CONIRD - Congresso Nacional de Irrigação e Drenagem, Uberaba -MG, 7p.

Souza, L. O. C., Mantovani, E. C. M., Soares, A. A., Ramos, M. \& Freitas, P. S. L. (2006). Avaliação de sistemas de irrigação por gotejamento, utilizados na cafeicultura. R. Bras. Eng. Agríc. Ambiental, 10(3), 541-548.

Wu, I. P. (1995). Optimal scheduling and minimizing deep seepage in microirrigation. Transactions of the ASAE, 38(5), $1385-1392$.

Zocoler, J. L. (2021). Avaliação de desempenho de sistemas de irrigação. UNESP. <http://www.agr.feis.unesp.br/irrigacao.html>. 